\section{Stroke patients benefit from early supported discharge services}

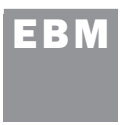

A recent meta-analysis has confirmed that early supported discharge (ESD) services, when properly managed, can reduce long-term dependency and hospital stays for stroke patients.

Peter Langhorne and an international group of colleagues used the Cochrane Stroke Group search strategy to identify appropriate randomized trials. Eleven studies-all of which compared conventional care with any ESD intervention for hospital inpatients with stroke-were included in the subsequent analysis. These studies provided data on a total of 1,597 patients, most of whom were diagnosed at city hospitals.

The results showed that the main outcomes of death or dependency were significantly less likely among patients who were assigned an ESD than in those assigned conventional care (odds ratio $0.79,95 \% \mathrm{Cl} 0.64-0.97, P=0.02$ ). In addition, ESD services were associated with an 8-day reduction in hospital stays $(P<0.0001)$. Although economic data were available for only five of the studies and were not collected in a uniform way, the analysis suggested that ESD services offered a cost saving relative to the equivalent in-hospital care.

In summary, the study showed that patients who receive ESD services after a stroke are more likely to be living independently at home than those who receive standard care, even when a dedicated stroke unit is available. Langhorne et al. recommend that ESD services should, therefore, be considered as part of a comprehensive stroke service.

Original article Langhorne P et al. (2005) Early supported discharge services for stroke patients: a meta-analysis of individual patients' data. Lancet 365: 501-506

\section{Ximelagatran: recent trial results}

Ximelagatran, a prodrug form of melagatran, is an oral direct thrombin inhibitor that promises to overcome some of the problems associated with other anticoagulants such as warfarin. Two papers published in JAMA now report on recent clinical trials of the drug. In the first study,
Fiessinger et al. investigate the use of ximelagatran for the prevention of recurrent venous thromboembolism, whereas the second paper describes its use in the prevention of stroke and systemic embolism in patients with atrial fibrillation.

Fiessinger et al. carried out the Thrombin Inhibitor in Venous Thromboembolism (THRIVE) treatment study, an international, randomized, double-blind noninferiority trial, to compare the safety and efficacy of fixed-dose ximelagatran with the standard treatment of enoxaparin (low-molecular-weight heparin) and adjusteddose warfarin, in patients with acute deep vein thrombosis. About a third of the 2,489 participants also had pulmonary embolism. During the 6-month treatment period, the rates of venous thromboembolism were similar in patients assigned to the ximelagatran or enoxaparin/ warfarin groups (estimated cumulative risks of $2.1 \%$ and $2.0 \%$, respectively), thus noninferiority of ximelagatran was demonstrated. The rates of major bleeding and mortality were also similar in the two groups. Serum alanine aminotransferase levels were abnormally high in $10 \%$ of patients receiving ximelagatran.

The Stroke Prevention using an Oral Thrombin Inhibitor in Atrial Fibrillation V (SPORTIF V) trial, a double-blind, randomized trial carried out at 409 sites in the US and Canada, compared fixed-dose ximelagatran with adjusted-dose warfarin in 3,922 patients with nonvalvular atrial fibrillation and additional risk factors such as hypertension, advanced age or systemic embolism. During the mean follow-up of 20 months, the rates of all strokes and systemic embolic events were similar in the two treatment groups (1.6\% vs $1.2 \%$ per year for the ximelagatran and warfarin groups, respectively), again demonstrating noninferiority of ximelagatran to standard treatment. Total bleeding was significantly less frequent among patients receiving ximelagatran, although the rates of major bleeding were similar in the two treatment groups. Again, elevated levels of serum alanine aminotransferase were observed with ximelagatran treatment, this time in $6 \%$ of patients.

In summary, these studies provide evidence of the efficacy of ximelagatran as an alternative to standard, warfarin-based treatments, in two different settings. As noted in an accompanying editorial by Victor Gurewich, the cost-effectiveness of treatment using this drug remains to be determined, and it will be 